\title{
Las metástasis óseas del cáncer
}

\section{Bone metastases}

\author{
S. Vicent ${ }^{1}$, D. Luis-Ravelo ${ }^{1}$, I. Antón ${ }^{1}$, I. Hernández ${ }^{1}$, S. Martínez ${ }^{1}$, J. de las Rivas², A. Gúr- \\ pide $^{3}$, F. Lecanda ${ }^{1}$
}

\section{RESUMEN}

Las metástasis óseas representan un problema clínico devastador en las neoplasias más frecuentes, especialmente en el mieloma múltiple, mama, próstata, y pulmón. Las consecuencias incluyen dolores refractarios a analgésicos convencionales, osteolisis que conlleva en ocasiones compresión medular, fracturas patológicas, y trastornos metabólicos. Recientes avances en el diagnóstico mediante técnicas de imagen, así como diversas técnicas bioquímicas, han favorecido un certero diagnóstico y seguimiento. El aumento de la supervivencia se ha mejorado mediante una aproximación multimodal en los tratamientos con la combinación de la inhibición de la osteolisis, la cirugía ortopédica profiláctica y la radioterapia. Recientes progresos en la investigación básica han determinado la huella molecular de metástasis de un tumor capaz de predecir su proclividad metastásica. La investigación básica favorecerá un conocimiento de los mecanismos básicos y llevará a elucidar dianas moleculares que favorecerán el desarrollo de fármacos capaces de prevenir, amortiguar o bloquear el proceso metastático.

Palabras clave. Tropismo. Osteolisis. Tratamiento.

\begin{abstract}
Bone metastases represent a devastating clinical problem in the most frequent neoplasies, especially in multiple myeloma, tumours breast, prostate and lung. The consequences include pain which is refractory to conventional analgesics, osteolysis often leading to bone-marrow compression and pathological fractures, and metabolic disorders. Recent advances in diagnosis using imaging techniques as well as different biochemical techniques have helped accurate diagnosis and follow-up. The increase in survival has improved through a multimodal approach combining, inhibition of osteolysis, with prophylactic orthopaedic surgery and radiation therapy. Recent advances in basic research have determined the molecular metastatic that can predict its proclivity to metastasize. Basic research will improve understanding of the basic mechanisms and lead to the clarification of molecular targets that will help in the development of medicines capable of preventing, decreasing or blocking the metastatic process.
\end{abstract}

Key words. Tropism. Osteolysis. Treatment.
1. División de Oncología, Laboratorio de Adhesión y Metástasis, Centro de Investigación Médica Aplicada (CIMA), Universidad de Navarra, Pamplona, Navarra.

2. CSIC y Universidad de Salamanca (CSIC/USAL). Campus Miguel de Unamuno. Salamanca.

3. Clínica Universitaria de Navarra.

Aceptado para su publicación el 8 de junio de 2006.

\section{Correspondencia:}

Fernando Lecanda

Laboratorio de Adhesión y Metástasis

Centro de Investigación Médica Aplicada

(CIMA)

Universidad de Navarra

31080 Pamplona

NAVARRA

Tfno. 948194700

Fax: 948194714

E-mail: flecanda@unav.es 


\section{UN PROBLEMA CLAVE EN CÁNCER: LA METÁSTASIS}

La metástasis representa una de las improntas que caracterizan a las células tumorales ${ }^{1}$, y consiste en la diseminación de las células de un tumor primario a órganos o tejidos distantes ${ }^{2,3}$. Constituye el aspecto más devastador del cáncer ${ }^{4}$. Se calcula que alrededor del $90 \%$ de los pacientes con cáncer mueren de metástasis ${ }^{5}$. Es pues una de las causas últimas de la elevada mortalidad del cáncer frente a la cual existen limitados recursos terapéuticos y clínicos.

El tropismo selectivo de las células metastásicas a ciertos órganos representa una de las singularidades más notables conocida como la hipótesis del "seed and soil" . Stephen Paget en 1889 estableció la siguiente observación: la diseminación del cáncer no es un proceso aleatorio sino que debe existir afinidad entre las células tumorales y el tejido diana de la misma forma que existe una compatibilidad entre "la semilla y la tierra fértil" . En definitiva, no todos los tumores metastatizan y lo hacen a los mismos órganos, sino que existe un patrón característico de metástasis de cada tumor a ciertos órganos. Es por tanto un proceso no aleatorio, aunque está altamente influido por el flujo sanguíneo y linfático tanto intra como peritumoral. Por ejemplo, el cáncer de pulmón forma predominantemente metástasis torácicas en ganglios linfáticos y cavidad pulmonar, y otros órganos entre los que se incluyen cerebro, hueso y glándulas suprarrenales ${ }^{8}$.

Desde un punto de vista celular, la metástasis ocurre como una etapa más dentro del proceso secuencial de la carcinogénesis ${ }^{2}$. El $90 \%$ de los tipos de cáncer poseen un origen epitelial y por ello la comprensión del desarrollo de metástasis se infiere del proceso de carcinogénesis epitelial. La metástasis es un proceso en el que tras el insulto genotípico, y la incapacidad por reparar el daño en el DNA, se produce una alteración estable en éste, que se traduce en cambios paulatinos de sus características fenotípicas y funcionales. Tras el aumento posterior disminución de la capacidad proliferativa y la motilidad celular, y la adhesión, tanto con las células vecinas, como con la membrana basal, se produce un desequilibrio funcional y estructural con el microentorno celular. En ese punto, gracias a la secreción de enzimas proteolíticas, una célula tumoral que ha perdido el anclaje celular es capaz de destruir la barrera de la membrana basal y el endotelio sanguíneo o linfático antes de migrar y ser transportada por los vasos a tejidos distantes. Estas etapas iniciales constituyen procesos ineficientes y la mayoría de las células no sobreviven en condiciones de independencia de anclaje. Para que se produzca la metástasis ósea se requiere que la célula una vez retenida en los sinusoides de la médula, migre y atraviese la pared para adherirse a la matriz extracelular de alguna superficie ósea del endostio, o periostio, donde es capaz de estimular a osteoblastos y osteoclastos. En muchas ocasiones, la capacidad de parada en un capilar, y finalmente la extravasación y crecimiento en el órgano diana no son necesarias, y basta que las células tumorales queden inmovilizadas en un capilar o retenidas como agregados plaquetarios para que puedan desarrollar un tumor secundario ${ }^{9-11}$.

No existe un patrón cronológico preciso de metástasis durante la carcinogénesis, y el escape de células del tumor primario puede llevarse a cabo casi simultáneamente en estadios muy tempranos del tumor primario. En este sentido, alrededor del $30 \%$ de pacientes en 
estadio I de cáncer de pulmón no microcítico mueren tras la resección completa del tumor, debido a la presencia de metástasis indetectables al diagnóstico ${ }^{12}$. En raras ocasiones el desarrollo del tumor secundario ocurre tras un tiempo de latencia de más de 10 años tras la remisión completa del tumor primario. Más frecuentemente, el aumento de metástasis se correlaciona con estadios avanzados del tumor primario. En la medida en que la carga tumoral aumenta se incrementan proporcionalmente las posibilidades de metástasis como consecuencia del aumento invasivo hacia tejidos y órganos circundantes.

\section{EL HUESO COMO ÓRGANO DIANA DE METÁSTASIS}

Una de las características propias tanto de tumores sólidos como hematológicos consiste en la frecuente propensión a formar metástasis óseas. Se ha estimado que anualmente mueren 350.000 personas con metástasis óseas en EEUU ${ }^{13}$. El hueso es el tejido diana de metástasis en el 95\% de los pacientes con mieloma múltiple, $75 \%$ de los pacientes con cáncer de mama y próstata y un $30-40 \%$ en los pacientes con cáncer de pulmón ${ }^{12,14}$ (Tabla 1).

Tabla 1. Incidencia y prognosis de las metástasis óseas de diversos tipos de cáncer.

\begin{tabular}{lccc}
\hline & $\begin{array}{c}\text { Incidencia } \\
\text { de enfermedad }\end{array}$ & $\begin{array}{c}\text { Mediana de } \\
\text { supervivencia (meses) }\end{array}$ & $\begin{array}{c}\text { Supervivencia a } \\
\mathbf{5} \text { años }\end{array}$ \\
\hline Mieloma & $95-100 \%$ & 20 & $10 \%$ \\
Mama & $65-75 \%$ & 24 & $20 \%$ \\
Próstata & $65-75 \%$ & 40 & $25 \%$ \\
Pulmón & $30-40 \%$ & $<6$ & $<5 \%$ \\
Riñón & $20-25 \%$ & 6 & $10 \%$ \\
Tiroides & $60 \%$ & 48 & $40 \%$ \\
Melanoma & $15-45 \%$ & $<6$ & $<5 \%$ \\
\hline
\end{tabular}

Datos de Rubens y Coleman.

Esta avidez por el tejido óseo se explica por un lado por la irrigación propia de los huesos, especialmente del compartimento medular donde resulta fácil que las células queden retenidas en los amplios sinusoides vasculares ${ }^{15}$. Por otro, los huesos albergan una gran variedad de tipos celulares y un medio rico en factores de crecimiento, citoquinas y quimoquinas que además de la atracción que ejercen sobre las células constituyen un medio adecuado para el crecimiento celular ${ }^{16}$. Además el propio tejido óseo constituye un almacén de diversos factores de crecimiento como IGF-1, FGF, PDGF y el mayor reservorio de TGF- $\beta$, un potente factor de crecimiento que regula multitud de procesos celulares ${ }^{17,18}$.

El tejido óseo se remodela continuamente para hacer frente a las demandas orgánicas de calcio y fósforo, y a las tensiones mecánicas. Esto se lleva a cabo por la acción coordinada de los osteoblastos, células encargadas de depositar matriz extracelular, y los osteoclastos, células de la línea monocito-macrófago con capacidad de producir resorción de la matriz mineralizada, favoreciendo un delicado balance. La llegada de células tumorales va a alterar ese equilibrio, 
favoreciendo un aumento de la resorción sobre la formación, produciendo las consiguientes lesiones osteolíticas. En ocasiones aumenta el número de ciclos de remodelado óseo y se produce junto a áreas de resorción, otras con un incremento de la formación ósea. Entre los tipos de tumores con mayor capacidad de metástasis a hueso se encuentran, el mieloma múltiple, mama y próstata (Tabla 1). También por la incidencia y la escasísima supervivencia destacan especialmente el cáncer de pulmón y en menor número el resto.

\section{MECANISMOS DE METÁSTASIS ÓSEAS}

Las metástasis a hueso pueden ser osteolíticas, las más frecuentes, y osteoblásticas, en las que las células tumorales inducen una formación ectópica de hueso reticular ${ }^{9,11,13,19}$.

La osteolisis parece estar mediada por factores derivados de las células tumorales que actúan directamente sobre el microentorno celular, o bien indirectamente activando la diferenciación y activación de los osteoclastos mediante la liberación de factores osteoclastogénicos. En el mieloma múltiple por ejemplo, las lesiones son puramente líticas inducidas por una activación exacerbada de osteoclastos adyacentes a las células tumorales mientras la formación ósea está totalmente inhibida ${ }^{20}$.

Las metástasis osteoblásticas son típicas del tumor de próstata, aunque también se han detectado en otros tumores de forma muy minoritaria (Fig. 1). La inducción de la formación de hueso ectópico y consiguiente osteosclerosis constituye un fenómeno notable cuyos mecanismos son poco conocidos.



Figura 1. Diferentes tipos de tumores producen diversos tipos de lesiones. A. Tumor de pulmón que ha producido metástasis osteolíticas; obsérvese la epífisis proximal humeral y la metáfisis distal. B. Carcinoma de próstata que ha producido una metástasis con formación de hueso ectópico, característica lesión osteoblástica. 
Las observaciones histológicas en biopsias establecen que suele ser más frecuente, sin embargo, la aparición de áreas óseas osteolíticas inducidas por el tumor y concomitantemente, áreas con activa osteoformación. Este espectro no resulta sorprendente puesto que la formación y resorción son procesos acoplados dentro del ciclo del remodelado óseo. En pacientes es frecuente observar simultáneamente aumento de trazadores óseos en las metástasis (por aumento de la actividad osteoclástica) y concurrentemente aumento de marcadores de la actividad osteoblástica.

\section{MÉTODOS DIAGNÓSTICOS DE LAS METÁSTASIS ÓSEAS}

Las técnicas diagnósticas más frecuentemente utilizadas incluyen la radiografía, la gammagrafía con tecnecio-99, la Tomografía Axial Computerizada (TAC) y la Resonancia Magnética Nuclear (RMN).

La radiografía por el bajo coste y la rapidez es el método de elección, a pesar de que posee una sensibilidad baja. La gammagrafía, sin embargo, presenta la ventaja de conseguir una imagen esquelética total, y se utiliza para el seguimiento de los tratamientos. La utilización como método de "screening" se ve limitado por la frecuencia de falsos positivos debidos a la activación de áreas osteoblásticas inducida por diversas enfermedades benignas.

Finalmente, la TAC o la RMN, por su elevado coste sólo se utilizan cuando existen dudas en el diagnóstico de las técnicas anteriores. Las imágenes topográficas de la TAC se utilizan para el estudio de lesiones pélvicas, y para lesiones corticales. Finalmente la RMN, debido a su gran resolución, se utiliza para el estudio de metástasis medulares.

Existen marcadores subrogados de metástasis a hueso que coinciden con los marcadores bioquímicos que se utilizan para evaluar el metabolismo óseo. Se pueden utilizar tanto para valorar la extensión como para el seguimiento del tratamiento aunque los resultados han sido hasta el momento variables. Los marcadores más utilizados son los niveles séricos de fosfatasa alcalina, y de hidroxiprolina y calcio urinario normalizados para la secreción de creatinina. Estos son marcadores poco específicos pudiendo verse alterados por múltiples factores. Los marcadores más específicos incluyen la valoración de los productos de degradación del colágeno tipo I en la orina o en el suero. Estos incluyen la determinación bioquímica de los niveles de N-telopéptido, y C-telopéptido séricos. Y finalmente los niveles de piridolina y desoxipiridolina en orina, marcadores de la tasa de resorción ósea, pueden servir para monitorizar la enfermedad ósea metastásica ${ }^{21-23}$. Como conclusión, la presencia de micrometástasis al diagnóstico constituye un elemento predictor de mal pronóstico de la enfermedad en el cáncer de mama ${ }^{24}$ por lo que actualmente se están desarrollando otros métodos como el uso de nanopartículas magnéticas se han usado para la detección de metástasis en ganglios linfáticos con $\mathrm{MRI}^{25}$.

\section{LIMITACIONES DE LOS TRATAMIENTOS ACTUALES}

La eficacia en el tratamiento de las metástasis óseas pasa por una aproximación multimodal donde se evalúen cautelosamente los beneficios y los riesgos para cada tipo de tumor, el estadio, extensión y naturaleza de la enfermedad metastásica.

En muchos casos, las metástasis óseas se caracterizan por ser refractarias a los tratamientos convencionales disponibles, por ello, 
los tratamientos se limitan a disminuir la morbilidad, y a paliar las consecuencias asociadas. Por tanto, es necesario desarrollar terapias eficaces capaces de incidir en el desarrollo tumoral.

\section{Cirugía ortopédica y traumatológica}

La osteolisis severa induce dolores agudos junto con una paulatina pérdida de las propiedades mecánicas del hueso (Fig. 1). Especialmente en aquellos huesos con gran carga mecánica, huesos de las extremidades inferiores, y del esqueleto axial, es importante valorar junto con el diagnóstico radiológico y gammagráfico la oportunidad de una intervención profiláctica, puesto que la estabilización ósea en esos casos facilita una rehabilitación y convalecencia eficaces. Especial mención merece el colapso vertebral, con la aparición de cifoescoliosis, restricción de la capacidad pulmonar y el peligro de la compresión medular que puede degenerar en una morbilidad y mortalidad notables. Los tumores más frecuentes que producen estas complicaciones son: mama, pulmón, próstata, linfoma y carcinoma renal. En general, la infiltración y/o radioterapia pueden disminuir los dolores asociados y aumentar el bienestar del paciente.

\section{Radioterapia y uso de radionúclidos sistémicos}

Es un método efectivo y rápido para aliviar el dolor ${ }^{26}$ en lesiones localizadas. La irradiación en extensas áreas puede producir fibrosis comprometiendo ulteriores tratamientos ${ }^{27}$.

De mayor eficacia terapéutica por la mayor selectividad hacia el tejido tumoral y menor toxicidad por su administración en un corto periodo de tiempo, también se utilizan el fósforo-32, el estroncio-89, el samario-153 y el renio-186. En las metástasis escleróticas de cáncer de próstata se utiliza con gran eficacia terapéutica el estroncio89 por su capacidad de fijarse las zonas de actividad osteoblástica ${ }^{28,29}$. También se ha utilizado en samario-153 conjugado con EDTMP en el cáncer de mama y próstata ${ }^{30}$.

\section{Bifosfonatos}

Poseen una estructura semejante a los pirofosfatos inorgánicos, se intercalan durante la formación de la hidroxiapatita. Posee una capacidad de resistencia a la osteolisis ácida mediada por los osteoclastos e impiden el anclaje robusto de los osteoclastos para formar la laguna de Howship, por su avidez por las integrinas $\alpha \mathrm{v} \beta 3 \mathrm{y} \beta 5$, bloqueando así la resorción ósea. Entre los bifosfonatos que se utilizan en el tratamiento de las metástasis óseas, el más potente es el ácido zoledrónico, con gran potencia antirresortiva, aunque también se utilizan otros como el clodronato e ibandronato. Sin embargo, el descubrimiento de algunos efectos secundarios del ácido zoledrónico recientemente descritos, la osteonecrosis mandibular ${ }^{31}$, ponen en entredicho el uso de este último, o el régimen terapéutico utilizado. Se han descrito otros efectos y mecanismos de estos compuestos que quedan al margen de esta revisión.

Poseen una eficaz acción disminuyendo de forma rápida la hipercalcemia de origen tumoral. Y son conocidos sus efectos analgésicos sobre una variedad de metástasis óseas. En algunos casos inducen una marcada apoptosis y una disminución de la capacidad invasiva 
de las células tumorales ${ }^{32-34}$. Una observación notable es la disminución de la carga tumoral como resultado del tratamiento con estos fármacos antiresortivos ${ }^{35}$. Estos efectos se potencian con la combinación de tratamientos estándar como la dexametasona en el mieloma múltiple, o al tamoxifeno y paclitaxel en el cáncer de mama. También se han descrito efectos antiangiogénicos por su capacidad de unión y bloqueo de las integrinas del endotelio vascular que también participan en este proceso ${ }^{36}$. Todo ello, facilita "in vivo" la disminución de la osteolisis inducida por el tumor en el caso del mieloma múltiple, cáncer de mama, próstata y otros tumores osteolíticos como el de pulmón ${ }^{37,38}$.

\section{EL FUTURO ES EL PRESENTE DE LA INVESTIGACIÓN BIOMÉDICA}

Al igual que en muchos otros aspectos del cáncer, el área de investigación en metástasis ha conocido importantes avances recientes especialmente en los relacionados con la biología celular y molecular implicadas en su desarrollo. Este progreso propiciado por la utilización del análisis masivo y sistemático de la expresión génica, han permitido a nivel experimental sentar las bases para la identificación de nuevos marcadores predictivos de metástasis en el tumor primario, con relevancia diagnóstica y pronóstica, abriendo así un camino expedito para el desarrollo de nuevas estrategias terapéuticas racionales, selectivas y eficientes.

Desde un punto de vista molecular la metástasis surge de cambios celulares sutiles que mediante un proceso de selección proporcionan ventajas celulares dentro de una población heterogénea. Estos cambios surgen propiciados por la inestabilidad cromosómica $\mathrm{y}$ vienen determinados por el despliegue de un programa genético progresivo que se induce por las interacciones heterotípicas de las células del tumor con el estroma, con las células del sistema inmune, la hipoxia, además de los cambios genéticos y epigenéticos del tumor $^{39}$. En la base de este proceso existen cambios coordinados de la expresión génica que modulan la actividad celular confiriendo progresivas ventajas -en la motilidad, adherencia y quimiotaxis- que favorecen el tropismo e invasividad de algunas células del tumor primario adquiriendo la capacidad metastásica. Esas ventajas adicionales sobre el resto permiten una segregación de las células y las capacitan para recorrer las consiguientes etapas secuenciales de la metástasis: la invasión de células desde el tumor primario a los vasos sanguíneos y linfáticos; la supervivencia en circulación; la adhesión a células endoteliales; la extravasación a órganos distantes; la colonización y el crecimiento en el tejido diana. Las interacciones heterotípicas entre las células tumorales y su microentorno celular, el flujo sanguíneo, y otros factores locales y sistémicos, modulan la diseminación de las células tumorales y el tropismo selectivo a diversos órganos.

Aunque algunos casos se ha demostrado en modelos experimentales la importancia de un único factor como iniciador de la metástasis, o involucrado en la invasión ósea, la traslación clínica ha sido escasa. Por ejemplo, cuando se estudió el papel del factor de transcripción "Twist", que había resultado clave en un modelo de cáncer de mama en ratones en muestras humanas, se observó una correlación reduci- 
da con un subtipo de estos tumores ${ }^{40}$. De forma similar, se puso de relieve la importancia de moléculas clave en el proceso de invasión como el eje VEGF-C y su receptor en diversos modelos animales de metástasis ${ }^{41}$, o la metaloproteasa-7 en un modelo animal de cáncer de próstata $^{42}$. Sin embargo, la correlación de estos factores en muestras clínicas ha sido limitada.

Como consecuencia de estos estudios parece clara la naturaleza multigénica implicada en la metástasis. En esta línea, se ha identificado el perfil molecular metastásico en una serie de diferentes tumores sólidos $^{43}$. Recientemente, un estudio mediante microarrays de expresión en mama comparando en un amplio panel de adenocarcinomas el tumor primario con la metástasis, se pudo determinar "la firma de mal pronóstico" que consistía en la expresión diferencial de un número reducido de genes ${ }^{4446}$. Estos genes son capaces de predecir el aumento de la invasividad y correlacionan con una limitada supervivencia. De esta forma, conociendo ese perfil genético en el tumor, el pronóstico clínico de pacientes de cáncer de mama puede predecirse. Sin embargo, este panel de genes de mal pronóstico no se corresponde necesariamente con los genes que son predictores de metástasis, -aunque algunos de ellos coincidan- ni con genes determinantes del tropismo órgano-específico. Más recientemente utilizando una estrategia de análisis masivo de la expresión génica mediante microarrys de expresión comparativo entre clones altamente metastásicos a hueso con el clon parental por sucesivas rondas de selección de poblaciones metastásicas "in vivo" en un modelo murino, Khan y $\mathrm{col}^{47,48}$ han identificado "la huella molecular del tropismo" del cáncer de mama a hueso. Estos experimentos evidencian que si bien en el tumor primario pueda existir una huella molecular de mal pronóstico, la expresión de un grupo de genes metastásicos debe inducirse para la consecución del fenotipo metastásico órgano-específico. Un trabajo de investigación reciente, Minn et al, consiguieron identificar una serie de genes mediadores de la metástasis del cáncer de mama a pulmón que fueron validados en muestras clínicas restrospectivas, prediciendo la capacidad de formar metástasis pulmonares ${ }^{49}$.

A la luz de estos estudios es por tanto predecible en los próximos años, mediante la utilización de la misma estrategia, que queden definidas las huellas predictivas de metástasis a distintos órganos-diana, incluido el hueso. Para ello es necesario, el desarrollo de nuevos modelos animales de xenotransplante ${ }^{50}$, que sirvan como plataformas experimentales para realizar estos estudios de investigación básica o translacional, donde se recapitule de manera fidedigna el desarrollo metastásico humano, así como las herramientas computacionales (bioinformática), para los análisis exhaustivos de los perfiles globales de expresión con plataformas transcriptómicas (microarrays de expresión) y proteómicas. Estos estudios constituyen la condición "sine qua non" para la identificación de genes marcadores, predictores, y mediadores del proceso metastásico que puedan constituir posteriormente dianas eficaces para el diseño de nuevos fármacos, o permitan la administración racional y selectiva de fármacos ya en desarrollo. El advenimiento de un futuro en el que el problema de la metástasis podrá ser tratado de forma eficaz farmacológicamente se hará realidad con una inversión coherente de la industria, junto con la sinergia del capital público, teniendo como horizonte indefectible la mejora de la salud y la calidad de vida de los pacientes. 


\section{BIBLIOGRAFÍA}

1. HANAHAN D, WeinBerg RA. The hallmarks of cancer. Cell 2000; 100: 57-70.

2. Chambers AF, Groom AC, MacDonald IC. Dissemination and growth of cancer cells in metastatic sites. Nat Rev Cancer 2002; 2: 563-572.

3. Woodhouse EC, ChuAqui RF, LiotTA LA. General mechanisms of metastasis. Cancer 1997; 80 (8 Suppl): 1529-1537.

4. Үокота J. Tumor progression and metastasis. Carcinogenesis 2000; 21 : 497-503.

5. Weigelt B, Peterse JL, VAN 'T Veer LJ. Breast cancer metastasis: markers and models. Nat Rev Cancer 2005; 5: 591-602.

6. PAget S. The distribution of secondary growths in cancer of the breast. Lancet 1889; 1: 571-573.

7. FIDLER IJ. The pathogenesis of cancer metastasis: the 'seed and soil' hypothesis revisited. Nat Rev Cancer 2003; 3: 453-458.

8. Ginsberg RJ KM, ARmstrong JG. Non-small cell lung cancer. Philadelphia, PA: Lippencott Williams and Wilkins, 2001.

9. GuISE TA, Mundy GR. Cancer and bone. Endocr Rev 1998; 19: 18-54.

10. Bogenrieder T, Herlyn M. Axis of evil: molecular mechanisms of cancer metastasis. Oncogene 2003; 22: 6524-6536.

11. Mundy GR. Mechanisms of bone metastasis. Cancer 1997; 80:1546-1556.

12. ColEMAN RE. Skeletal complications of malignancy. Cancer 1997; 80: 15881594.

13. Mundy GR. Metastasis to bone: causes, consequences and therapeutic opportunities. Nat Rev Cancer 2002; 2: 584-593.

14. Mohla S, Weilbacher KN, Cher ML, Oyajobi BO, Poznak CV, Clohisy DR. Third North American Symposium on Skeletal Complications of Malignancy: summary of the scientific sessions. Cancer 2003; 97: 719-725.

15. Kahn D, Weiner GJ, Ben-Haim S, Ponto LL, Madsen MT, Bushnell DL et al. Positron emission tomographic measurement of bone marrow blood flow to the pelvis and lumbar vertebrae in young normal adults. Blood 1994; 83: 958-963.

16. Mundy GR, Chen D, Zhao M, Dallas S, Xu C, HaRris S. Growth regulatory factors and bone. Rev Endocr Metab Disord 2001; 2:105-115.

17. Hauschka PV, Chen TL, Mavrakos AE. Polypeptide growth factors in bone matrix. Ciba Found Symp 1988; 136: 207-225.

18. Hauschka PV, Mavrakos AE, Iafrati MD, Doleman SE, Klagsbrun M. Growth factors in bone matrix. Isolation of multiple types by affinity chromatography on heparin-Sepharose. J Biol Chem 1986; 261: 12665-12674.

19. Roodman GD. Mechanisms of bone metastasis. N Engl J Med 2004; 350: 1655-1664.

20. Taube T, Beneton MN, McCloskey EV, Rogers S, Greaves M, Kanis JA. Abnormal bone remodelling in patients with myelomatosis and normal biochemical indices of bone resorption. Eur J Haematol 1992; 49: 192-198.

21. Costa L, Demers LM, Gouveia-Oliveira A, Schaller J, Costa EB, De Moura MC et al. Prospective evaluation of the peptide-bound collagen type I crosslinks N-telopeptide and C-telopeptide in predicting bone metastases status. J Clin Oncol 2002; 20: 850-856.

22. Tamada T, Sone T, Tomomitsu T, Jo Y, Tanaka H, Fukunaga M. Biochemical markers for the detection of bone metastasis in patients with prostate cancer: diagnostic efficacy and the effect of hormonal therapy. J Bone Miner Metab 2001; 19: 45-51.

23. Maeda H, Kolzumi M, Yoshimura K, Yamauchi T, Kawai T, Ogata E. Correlation between bone metabolic markers and bone scan in prostatic cancer. J Urol 1997; 157: 539-543. 
24. Braun S, Vogl FD, Naume B, Janni W, Osborne MP, Coombes RC et al. A pooled analysis of bone marrow micrometastasis in breast cancer $\mathrm{N}$ Engl J Med 2005; 353: 793-802.

25. Harisinghani MG, Barentsz J, Hahn PF, Deserno WM, Tabatabaei S, van de KaA $\mathrm{CH}$ et al. Noninvasive detection of clinically occult lymph-node metastases in prostate cancer. N Engl J Med 2003; 348: 2491-2499.

26. Tong D, GILLICK L, HendRICKSON FR. The palliation of symptomatic osseous metastases: final results of the Study by the Radiation Therapy Oncology Group. Cancer 1982; 50: 893-899.

27. JANJAN NA. Radiation for bone metastases: conventional techniques and the role of systemic radiopharmaceuticals. Cancer 1997; 80: 1628-1645.

28. Lewington VJ, McEwan AJ, Ackery DM, Bayly RJ, Keeling DH, Macleod PM et al. A prospective, randomised double-blind crossover study to examine the efficacy of strontium-89 in pain palliation in patients with advanced prostate cancer metastatic to bone. Eur J Cancer 1991; 27: 954-958.

29. Robinson RG, Preston DF, BAXter KG, Dusing RW, SPicer JA. Clinical experience with strontium- 89 in prostatic and breast cancer patients. Semin Oncol 1993; 20: 44-48.

30. Resche I, Chatal JF, Pecking A, Ell P, Duchesne G, Rubens R et al. A dose-controlled study of $153 \mathrm{Sm}$-ethylenediaminetetramethylenephosphonate (EDTMP) in the treatment of patients with painful bone metastases. Eur $\mathrm{J}$ Cancer 1997; 33: 1583-1591.

31. Woo SB, Hande K, RichaRdson PG. Osteonecrosis of the jaw and bisphosphonates. N Engl J Med 2005; 353: 99-102; discussion 99-02.

32. Senaratne SG, Pirianov G, Mansi JL, Arnett TR, Colston KW. Bisphosphonates induce apoptosis in human breast cancer cell lines. Br J Cancer 2000; 82: 1459-1468.

33. GREEN JR, ClezARdin P. Mechanisms of bisphosphonate effects on osteoclasts, tumor cell growth, and metastasis. Am J Clin Oncol 2002; 25: S3-9.

34. Rogers MJ, Gordon S, Benford HL, Coxon FP, LuCKMAn SP, MonkKonen J et al. Cellular and molecular mechanisms of action of bisphosphonates. Cancer 2000; 88: 2961-2978.

35. MuNDY GR. Bisphosphonates and tumor burden. J Clin Oncol 2002; 20: 3191-3192.

36. Pluijm G, LowiK C, PAPapoulos S. Tumour progression and angiogenesis in bone metastasis from breast cancer: new approaches to an old problem. Cancer Treat Rev 2000; 26: 11-27.

37. Rosen LS, Gordon D, TCheKMEDyian NS, Yanagihara R, HiRsh V, KRZAKowsKi M et al. Long-term efficacy and safety of zoledronic acid in the treatment of skeletal metastases in patients with nonsmall cell lung carcinoma and other solid tumors: a randomized, Phase III, double-blind, placebo-controlled trial. Cancer 2004; 100: 2613-2621.

38. Rosen LS, Gordon D, TCheKmedyian S, YanagiHaRa R, HiRsh V, KRZAKowsKi M et al. Zoledronic acid versus placebo in the treatment of skeletal metastases in patients with lung cancer and other solid tumors: a phase III, doubleblind, randomized trial-the zoledronic acid Lung Cancer and Other Solid Tumors Study Group. J Clin Oncol 2003; 21: 3150-3157.

39. HyNES RO. Metastatic potential: generic predisposition of the primary tumor or rare, metastatic variants-or both? Cell 2003; 113: 821-823.

40. Yang J, Mani SA, Donaher JL, Ramaswamy S, Itzykson RA, Come C et al. Twist, a master regulator of morphogenesis, plays an essential role in tumor metastasis. Cell 2004; 117: 927-939.

41. Su JL, Yang PC, Shin JY, Yang CY, Wei LH, Hsien CY et al. The VEGF-C/Flt-4 axis promotes invasion and metastasis of cancer cells. Cancer Cell 2006; 9: 209-223. 
42. Lynch CC, Hikosaka A, Acuff HB, Martin MD, KaWAi N, Singh RK et al. MMP-7 promotes prostate cancer-induced osteolysis via the solubilization of RANKL. Cancer Cell 2005; 7: 485-496.

43. Ramaswamy S, Ross KN, LANDER ES, Golub TR. A molecular signature of metastasis in primary solid tumors. Nat Genet 2003; 33: 49-54.

44. VAN 'T VeER LJ, Dai H, van de ViJver MJ, He YD, HaRt AA, MaO M et al. Gene expression profiling predicts clinical outcome of breast cancer. Nature 2002; 415: 530-536.

45. VAN DE ViJVER MJ, He YD, van't VeEr LJ, Dai H, HaRT AA, Voskuil DW et al. A gene-expression signature as a predictor of survival in breast cancer. $\mathrm{N}$ Engl J Med 2002; 347: 1999-2009.

46. VAN 'T VeER LJ, DAi H, VAN DE ViJver MJ, He YD, HaRt AA, Bernards R et al. Expression profiling predicts outcome in breast cancer. Breast Cancer Res 2003; 5: 57-58.

47. Kang Y, Siegel PM, Shu W, Drobnjak M, Kakonen SM, Cordon-Cardo C et al. A multigenic program mediating breast cancer metastasis to bone. Cancer Cell 2003; 3: 537-549.

48. Minn AJ, Kang Y, Serganova I, GuPta GP, GiRi DD, Doubrovin M et al. Distinct organ-specific metastatic potential of individual breast cancer cells and primary tumors. J Clin Invest 2005; 115: 44-55.

49. Minn AJ, Gupta GP, Siegel PM, Bos PD, Shu W, GiRi DD et al. Genes that mediate breast cancer metastasis to lung. Nature 2005; 436: 518-524.

50. Rosol TJ, TAnnehill-Gregg SH, LeRoy BE, Mandl S, Contag CH. Animal models of bone metastasis. Cancer 2003; 97: 748-757. 
